\title{
INFLUÊNCIA DA VARIAÇÃO DOS PARÂMETROS CLIMÁTICOS NA MODELAGEM DE SWTA: ESTUDO DE CASO BACIA HIDROGRÁFICA DO RIO TAPAJÓS
}

\author{
Edinelson Saldanha Correa'; David Gibbs Magrath²; Celso Bandeira de Melo Ribeiro³. \\ ${ }^{1}$ Universidade Federal do Oeste do Pará (UFOPA), Santarém, Pará, Brasil, edinelson_saldanha@hotmail.com \\ ${ }^{2}$ Universidade Federal do Oeste do Pará, Santarém, Pará, Brasil \\ ${ }^{3}$ Universidade Federal de Juiz de Fora, Juiz de Fora, Minas Gerais, Brasil
}

RESUMO: As mudanças no uso e ocupação do solo, têm causado modificações sistemas hidrológicos. Dessa forma, o conhecimento prévio do efeito das variações no uso do solo sobre a dinâmica das águas em bacias hidrográficas, são importantes para prever, mitigar e/ou eliminar atividades e intervenções com impactos negativos. Neste contexto, os modelos hidrológicos são importantes ferramentas para estas previsões. O presente trabalho teve como objetivo mostrar se a modelagem em SWAT é capaz de simular de forma satisfatória as vazões na bacia do rio Tapajós, confrontando os dados obtidos através das simulações com as vazões observadas para o período de 1999 a 2002, avaliando ainda como diferentes entradas de parâmetros climáticos afetam as simulações. Como entradas da modelagem foram usados três pontos fluviométricos Acará dos Tapajós, Fazenda Tratex e Jardim do Ouro; pedologia fornecida pela Empresa Brasileira de Pesquisa Agropecuária (EMBRAPA); uso e ocupação do solo foram realizados através da classificação supervisionada de 32 imagens Landsat (Janeiro/2001 a dezembro/2001); o modelo digital de elevação (MDT) utilizando imagens do Shuttle Radar Topography Mission (SRTM); quanto aos parâmetros climáticos, duas origens foram usadas a do Instituto Nacional de Meteorologia (INMET) e Global Precipitation Climatology Project (GPCP). Na simulação usando dados climáticos INMET, foram obtidos valores de $\operatorname{COE}(0.81,0.86$ e 0.79$)$ considerados muito bons e superiores quando comparados com os resultados das simulações usando entradas climáticas do GPCP. Mostrando assim que o modelo possui boa correlação com os dados reais, portanto, representando o comportamento hidrológico da bacia do rio Tapajós.

PALAVRAS-CHAVE:Modelagem hidrológica, Uso do solo, Vazão.

\section{INFLUENCE OF THE VARIATION OF THE CLIMATIC PARAMETERS IN THE MODELLING OF SWTA: STUDY OF CASE HYDROGRAPHIC BASIN OF THE RIVER TAPAJÓS}

ABSTRACT: Changes in use and occupation of the soil have caused modifications in
hydrological systems. Thus, prior knowledge on the effect of variations in the use of the soil
and the dynamics of waters in the river basins is important to predict, mitigate and/or
eliminate activities and interventions with negative impacts. In this context, hydrological
models are important tools for these predictions. This work aimed at showing if the SWAT
modeling is able of simulating satisfactorily the flows in the basin of Tapajós river, comparing

Agroecossistemas, v. 9, n. 1, p. 84-98, 2017, ISSN online 2318-0188 
the data obtained through the simulations with the flows observed during the period from 1999 to 2002. It also verified how different inputs of climatic parameters affect the simulations. As modeling inputs, three fluviometric points were used: Acará dos Tapajós, Fazenda Tratex and Jardim do Ouro; Pedology provided by the Brazilian Agricultural Research Corporation (EMBRAPA); use and occupation of the soil performed through the supervised classification of 32 Landsat images ( (January 2001 to December 2001); The digital elevation model (MDT) using Shuttle Radar Topography Mission (SRTM) images; Regarding the climatic parameters, two sources were used: National Institute of Meteorology (INMET) and Global Precipitation Climatology Project (GPCP). In the simulation with INMET climatic data, COE values were obtained $(0.81,0.86$ and 0.79$)$ and they were considered very good and higher compared with the results of the simulations with GPCP weather inputs. The work show, thus, that the model has a good correlation with the real data and represents the hydrological behavior of the basin of Tapajós river.

KEYWORDS: Flow, Hydrological modelling, Variations in soil use.

\section{INFLUENCIA DE LA VARIACIÓN DE LOS PARÁMETROS CLIMÁTICOS EN EL MODELADO DE SWTA: ESTUDIO DE CASO CUENCA HIDROGRÁFICA DEL RÍO TAPAJÓS}

RESUMEN: Los cambios en el uso y ocupación del suelo, han causado modificaciones sistemas hidrológicos. De esa forma, el conocimiento previo del efecto de las variaciones en el uso del suelo, sobre la dinámica de las aguas en cuencas hidrográficas, son importantes para prever, mitigar y/o eliminar actividades e intervenciones con impactos negativos. En este contexto, los modelos hidrológicos son importantes herramientas para estas previsiones. El presente trabajo tuvo como objetivo mostrar si el modelado en SWAT es capaz de simular de forma satisfactoria las vazões en la cuenca del río Tapajós, confrontando los datos obtenidos a través de las simulaciones con las vazões observadas para el periodo de 1999 a 2002, evaluando aún como diferentes entradas de parámetros climáticos afectan las simulaciones. Como entradas del modelado fueron usados tres puntos fluviométricos Acará de los Tapajós, Hacienda Tratex y Jardín del Oro; pedologia suministrada por la Empresa Brasileña de Investigación Agropecuaria (EMBRAPA); uso y ocupación del suelo fueron realizados a través de la clasificación supervisada de 32 imágenes Landsat (Enero/2001 a diciembre/2001); el modelo digital de elevación (MDT) utilizando imágenes del Shuttle Radar Topography Mission (SRTM); en cuanto a los parámetros climáticos, dos orígenes fueron usadas a del Instituto Nacional de Meteorología (INMET) y Global Precipitation Climatology Project (GPCP). En la simulación usando datos climáticos INMET, fueron obtenidos valores de COE (0.81, 0.86 y 0.79) considerados muy buenos y superiores cuando comparados con los resultados de las simulaciones usando entradas climáticas del GPCP. Mostrando así que el modelo posee buena correlación con los datos reales, por lo tanto, representando el comportamiento hidrológico de la cuenca del río Tapajós.

PALABRAS CLAVE: Modelado hidrológica, Simulación, Uso del suelo, Vazão. 


\section{INTRODUÇÃO}

Um dos grandes debates mundiais do século XXI é a questão hídrica, no que diz respeito à falta do recurso água. Este problema já assola a grande maioria dos países africanos e começa a afetar os países europeus (RUHOFF, 2004).

O Brasil, no entanto, habituou-se a ilusão que tal problema não o afetaria, por ser considerado uma das grandes potências hídricas do planeta. Todavia, em 2014, o estado de São Paulo começa a sofrer com a escacasses de água, tendo que fazer racionamento do recurso, tal realidade também abrangeu muitos municípios do estado do Pará, que mesmo sendo banhado pelos Rios Amazonas e Tapajós sofrem com a disponibilidade hídrica, não no que se refere a quantidade, mas quanto à distribuição e qualidade da água.

Atualmente, sabe-se que o uso dado ao solo, em conjunto com as características geomorfológicas da área de abrangência em uma bacia hidrográfica, é que definirá seu comportamento frente a eventos hidrológicos, como a precipitação, escoamento superficial e subterrâneo, evapotranspiração e infiltração, podendo indicar, por exemplo, uma maior ou menor tendência a enchentes (COSTA, 2007).

A conversão das florestas em pastos, campos de soja, agricultura ou mesmo a extração madeireira tem sérios custos sociais e ambientais. Entre os custos ambientais tem-se a mudança nos parâmetros do ciclo hidrológico e no balanço hídrico local ou regional, podendo-se citar ainda os efeitos nocivos dos agrotóxicos no meio-ambiente e na saúde humana; a expulsão das comunidades nativas de suas terras e; a erosão do solo com consequente assoreamento de mananciais. Seus danos são diversos e complexos, ocasionando problemas em efeito dominó (desmatamento, erosão e assoreamento) e em grande parte, possivelmente irreversíveis (ARAÚJO, 2010).

É inquestionável a importância econômica e social das diferentes atividades degradadoras do meio ambiente, entretanto, com isso se torna necessário conhecer qual o limite da conversão das áreas de florestas em áreas agropecuárias, mineração ou hidrelétricas, sem comprometer o equilíbrio dos ecossistemas naturais. 
Neste contexto, a modelagem surge como alternativa para a obtenção de informações sobre a dinâmica do comportamento, antecipação de eventos e previsão de possíveis impactos ambientais em uma bacia hidrográfica que seja de correntes de eventuais mudanças no uso da terra. Os modelos hidrológicos constituem instrumentos importantes para representar os sistemas, seus processos e para auxiliar na compreensão dos fenômenos que envolvem as variáveis do ciclo da água (RODRIGUES, 2015).

Por meio da modelagem hidrológica é possível estimar cenários futuros de uso e ocupação do solo e, consequentemente a demanda por recursos naturais, avaliando o grau de comprometimento dos recursos da bacia em foco. Ressaltase ainda que o uso da modelagem a partir da inserção de diferentes cenários de uso e manejo do solo pode contribuir para o planejamento do uso racional dos recursos hídricos e, posteriormente fornecer suporte à implementação de práticas conservacionistas para um desenvolvimento sustentável (DURÃES, 2010).

Diante disso os modelos hidrológicos vêm sendo cada vez mais utilizados, configurando-se como instrumentos técnicos na gestão de bacias hidrográficas, entre eles destaca-se o modelo de Soiland Water Assessment Tool (SWAT) que é utilizado para modelar diferentes processos físicos em bacias hidrográficas com o objetivo de estudar alterações no uso do solo e suas consequências nos escoamentos superficial e subterrâneo, na produção de sedimentos e na qualidade da água (SRINIVASAN et al., 1994).

Para reproduzir as condições reais da bacia, o modelo SWAT necessita de informações sobre as características físicas e climáticas. Com esse conjunto de variáveis, 0 modelo faz a compartimentação da bacia baseado no Modelo Digital do Terreno (MDT) que representa as condições topográficas de declividade e as formas do relevo, associadas às informações pedológicas e de uso e cobertura do solo. As sub-bacias são divididas em Unidades de Resposta Hidrológica (HRU), mantendo os parâmetros espacialmente distribuídos com características homogêneas, que representam a heterogeneidade dos fatores que influenciam o comportamento 
hidrológico (RODRIGUES, 2015).

O SWAT, em seu desenvolvimento, foi incorporando várias características contidas em modelos anteriores. O modelo tem por objetivo prever o efeito das ações de uso e manejo do solo sobre os recursos hídricos, produção de sedimentos, produção de nutrientes e pesticidas, sendo aplicado em pequenas e grandes bacias. A modelagem pode ser efetuada em intervalos de tempo diários, mensais e anuais.

Conforme Santos et al. (2005), a característica principal do SWAT é que o modelo que possui base física, com equações que descrevem a relação entre as variáveis do sistema. Requer como inputs informações sobre clima, solos, relevo, vegetação e uso e manejo do solo. Através destas informações, o SWAT modela os processos físicos associados com o movimento da água, movimento de sedimentos, crescimento da vegetação, ciclagem de nutrientes e qualidade da água. É distribuído, onde a bacia hidrográfica pode ser subdividida em sub-bacias de modo a refletir as diferenças de tipo de solo, cobertura vegetal, topografia e uso do solo; para as funções mais básicas, o modelo utiliza informações de fácil acesso, disponíveis em agências governamentais; aceita simular longos períodos de forma contínua, o que permite perceber impactos ambientais que só são visíveis quando avaliados por um longo período de tempo.

Para a simulação, o modelo requer informações espaciais, como o modelo digital do terreno, hidrografia, mapa de solos, mapa de uso do solo e de séries temporais de precipitação, vazão líquida, descarga sólida, temperatura mínima e máxima, radiação solar, umidade relativa e velocidade do vento.

Com relação aos solos, o modelo requer um banco de dados com diversas informações, como o número de camadas do solo, grupo hidrológico e informações por camada de solo, como capacidade de água disponível, condutividade hidráulica saturada e porosidade.

Baseado no exposto até aqui este trabalho tem como objetivo avaliar os resultados gerados na modelagem da vazão da bacia hidrográfica do rio Tapajós, usando duas diferentes entradas de dados climáticas (GPCP e INMET), no período de 1999 a 2002, utilizando índices de COE e PBIAS como mensuradores quantitativos. 


\section{MATERIAL E MÉTODOS}

O modelo hidrológico SWAT foi utilizado para simular as vazões entre os anos de 1999 a 2002, no rio Tapajós, este período foi adotado pois possui a mesma série temporal nas três estações fluviométricas adotadas neste trabalho. 0 modelo foi desenvolvido pelo USDA Agricultural Research Service (USDA-ARS) e pelo Texas A\&M AgrilifeResearch (ARNOLD et al., 2000).

O modelo hidrológico foi aplicado na sub-bacia do rio Tapajós (Figura 1). Segundo o Ministério do Meio Ambiente - MMA (2011), a bacia hidrográfica do Rio Tapajós abrange três Estados: Pará, Mato Grosso e Amazonas. Situa-se entre os paralelos $02^{\circ}$ e $15^{\circ}$ de latitude sul e os meridianos $54^{\circ}$ e $60^{\circ}$ de longitude oeste. Sua extensão territorial ocupa uma área de aproximadamente $493.000 \mathrm{~km}^{2}$.

Os dados meteorológicos (umidade relativa, radiação solar, velocidade do vento, temperatura) foram obtidos a partir de 4 estações meteorológicas, a localização das estações é apresentada na Figura 1, selecionadas segundo os critérios proposto por Pontes e Fernandes (2002), que propunha que a obtenção de resultados dos processos de simulação, satisfatórios às escolhas das estações, devem ter séries de dados pluviométricos superior a 10 anos, exigência também do modelo. Através dessas estações, foi possível calcular a estatística necessária para o modelo, para isso duas origens de entradas de dados foram usadas: INMET e GPCP.

Figura 1. Mapa de localização geral e das estações meteorológicas.

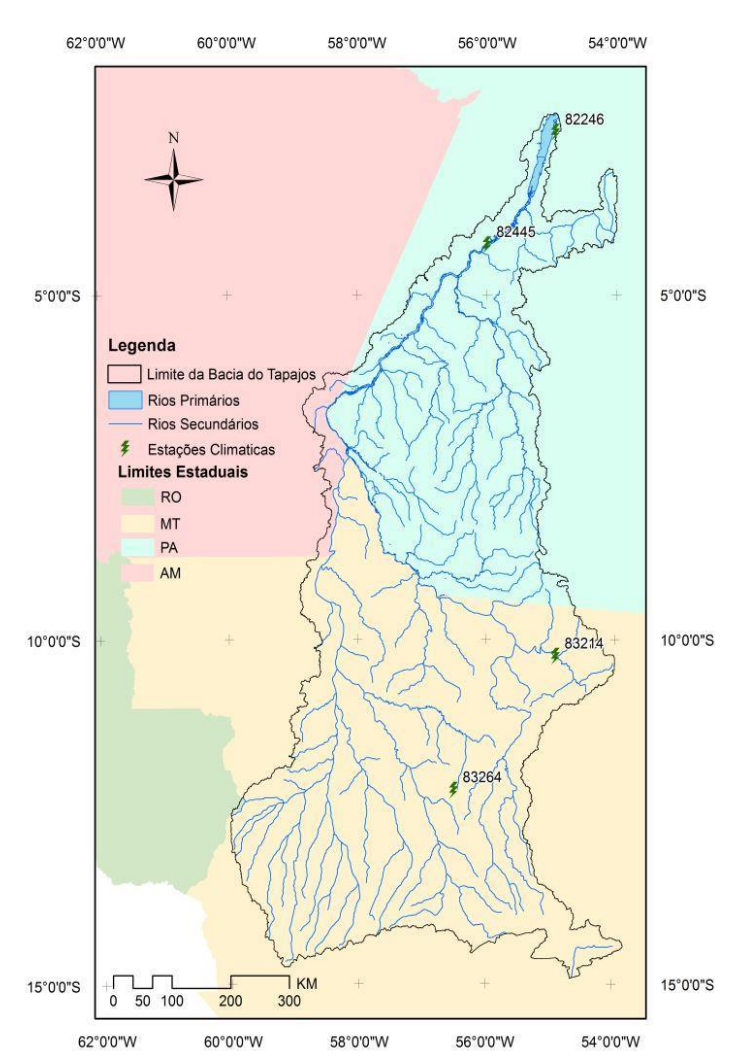

Fonte: Elaborado pelo autor (2017).

O Modelo digital de elevação: a área da bacia do rio Tapajós foi discretizada em células de área igual a $1.800 \mathrm{~m}^{2}(90 \mathrm{~m}$ x 90m - 1,8 hectares) utilizando imagens do Shuttle Radar Topography Mission 
(SRTM), formando assim uma matriz de células sobre a bacia. A escolha da área da célula foi definida de forma a captar a resolução espacial máxima correlata com a qualidade das informações planialtimétricas do modelo numérico do terreno (LIBOS, 2002).

O levantamento de pedológico na bacia rio Tapajós realizado pela EMBRAPA (2013), encontra-se exposto na Figura 2, inventariou os seguintes tipos: Solos Aluviais (AE) representado apenas 0,70\%; Areias Quartzosas (AQ) 18,16\%; Afloramento de Rochas (AR) 0,31\%; Cambissolo (C) 0,60\%; Gleissolo (G) 0,94\%; Latossolo Amarelo (LA) 5,86\%; Latossolo Vermelho-Escuro (LE) 6,64\%; Latossolo Vermelho-Amarelo (LV) 27,45\%; Plintossolo (PT) 0,06\%; Argissolo (AG) 33,15\%; Solos Litolicos (R) 5,14\%; Solos Petroplinticos (SP) 0,83\% e Terra Roxa Estruturada (TR) 0,17\%. As características do físico hídricas do solo foram obtidas de funções de pedotransferência (SAXTON; RAWLS, 2006; RIBEIRO et al., 2014) e nos resultados de trabalhos apresentados por Baldissera (2005), Silva (2015) e Lima (2013).
Figura 2 - Mapa de Classes Pedológicas

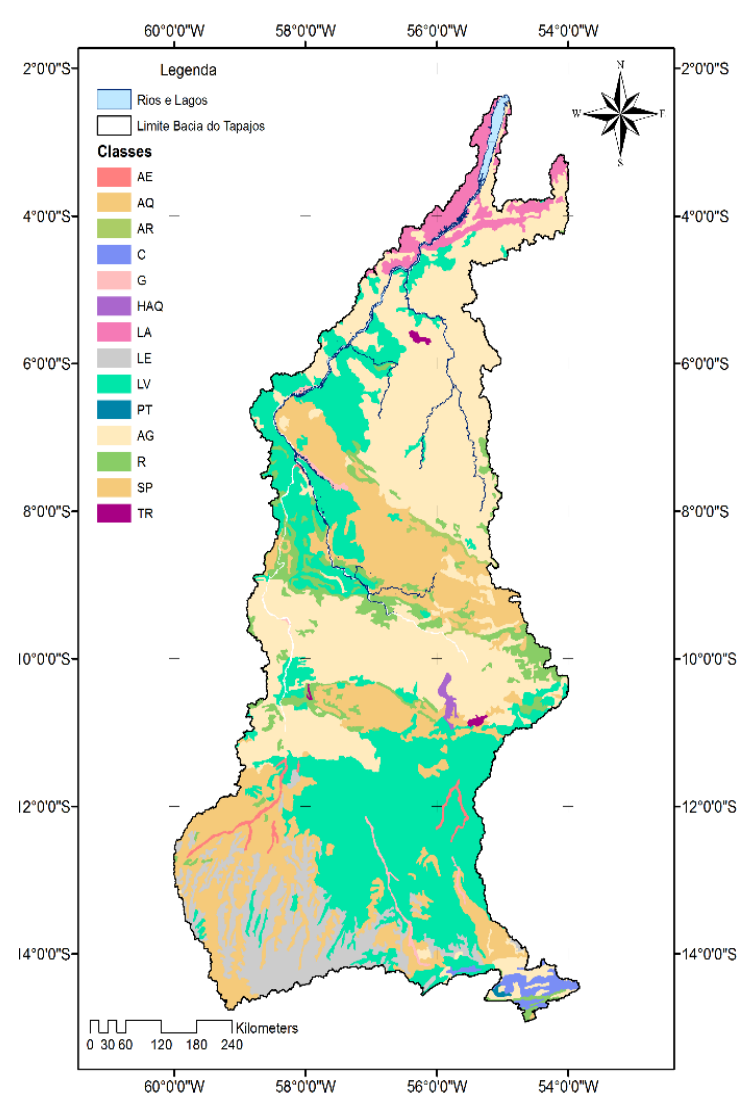

Fonte: Elaborado pelo autor (2017).

A investigação visual detalhada e a classificação supervisionada das trinta e duas imagens Landsat 7 (Janeiro/2001 a dezembro/2001), as quais, foram agrupadas em sete classes de uso e ocupação do solo: rios e lago, floresta, pastagens, savanas, áreas agrícolas, áreas urbanas e solo exposto, a distribuição espacial é ilustrada na Figura 3. Esta classificação foi baseada na classificação de uso e ocupação do solo da proposta pela United States Geological Survey (USGS), pois é uma das opções de banco de dados dispostas no SWAT. 
Figura 3 - Mapa de uso e ocupação do solo.

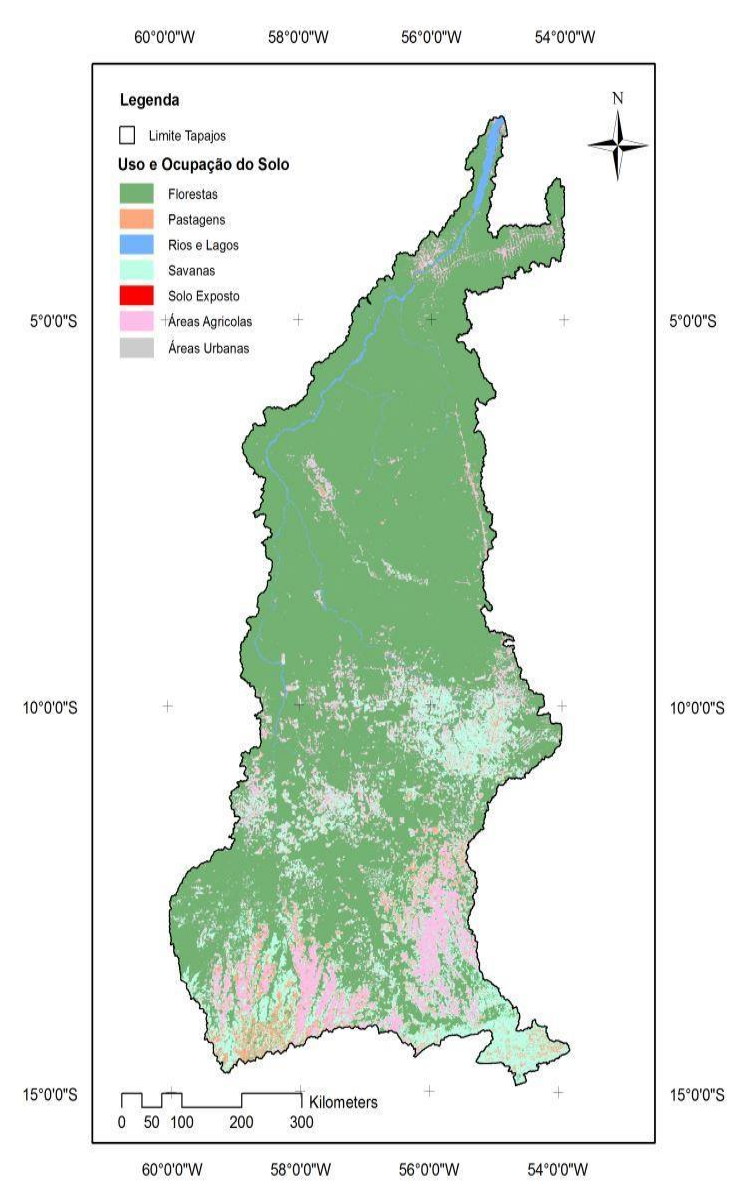

Fonte: Elaborado pelo autor (2017).

Os resultados da simulação foram importados pelo SWAT-CUP, onde foi possível fazer uma análise estatística e comparar os dados simulados com os observados. Foi possível, também, verificar os parâmetros mais sensíveis, o que permitiu calibrar e validar o modelo, de maneira a prever, de forma mais acurada, a vazão.

modelo foi utilizado para simular a vazão, iniciando com o período de aquecimento, que tem a finalidade de estabilizar o modelo durante seus processos interativos iniciais para a obtenção dos resultados. As incertezas no resultado do modelo, nas condições iniciais, são minimizadas através da utilização desse período que permite reduzir os eventuais erros nessas condições (WAGENER et al., 2004). Neste trabalho foi adotado um período de aquecimento para o modelo de 19 anos, de 01/01/1979 a 01/01/1998.

A calibração de um modelo hidrológico é um meio de se encontrar os valores dos parâmetros do modelo que melhor representem o comportamento hidrológico do sistema (COLLISHONN, 2008). Para isso as calibrações foram realizadas tomando como base três estações fluviométricas: Acará dos Tapajós, Fazenda Tratex e Jardim do Ouro, ilustradas na Figura 4, no período de janeiro de 1999 a dezembro de 2000, e as validações de janeiro de 2001 a dezembro de 2002. A partir dos dados observados nas estações fluviométricas de monitoramento e dos dados simulados pelo modelo, foi realizada a calibração, ainda em estágio preliminar, visando adequar o modelo ao comportamento hidrológico do local. 
Figura 4 - Mapa distribuição das subbacias no rio Tapajós.

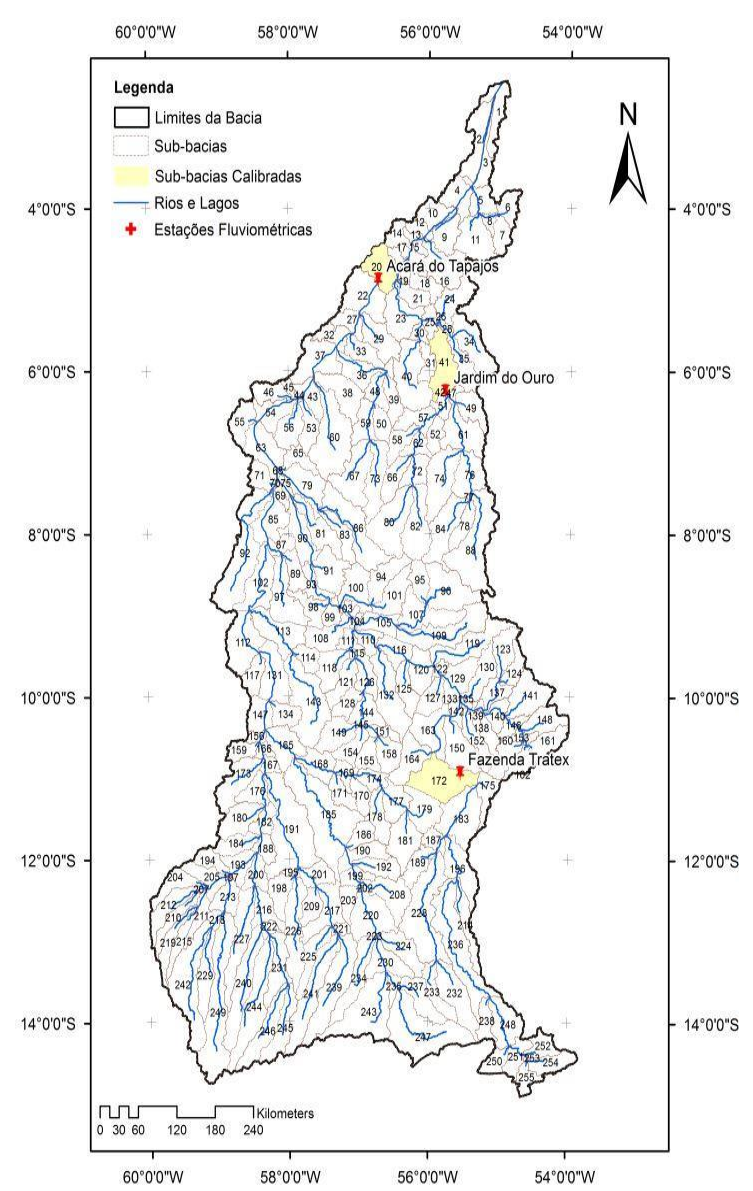

Fonte: Elaborado pelo autor (2017).

As simulações de vazão foram avaliadas utilizando diferentes índices de análises estatísticas para comparar dados observados com dados simulados. Estas análises são de extrema importância, pois é uma forma de verificar a eficiência da simulação. Neste trabalho foram utilizados os índices de eficiência de Nash e Sutciffe (COE) (NASH; SUTCLIFFE, 1970) e da porcentagem BIAS (PBIAS).

O índice de eficiência de NashSutcliffe foi utilizado com a finalidade de avaliar a eficiência do modelo em simular determinada variável. O COE pode variar a partir de $-\infty$ a 1 , sendo 1 indicativo de um perfeito ajuste dos dados simulados em relação aos dados medidos:

$$
C O E=1-\frac{\sum_{i=1}^{n}\left(Y_{i}^{o b s}-Y_{i}^{s i m}\right)^{2}}{\sum_{i=1}^{n}\left(Y_{i}^{o b s}-Y_{m}^{o b s}\right)^{2}}
$$

Onde:

yobs. é o evento observado;

Ysim. é o evento simulado;

$Y_{m}^{o b s}$ : é a média do evento observado no período de simulação;

n: é o número de eventos.

A PBIAS avalia a tendência geral que os dados simulados mostram em subestimar (valores PBIAS positivos) ou superestimar (valores PBIAS negativos) OS dados medidos (GUPTA et al., 1999):

$$
P B I A S=\sum_{i=1}^{n} \frac{\left(Y_{i}^{o b s}-Y_{i}^{s i m}\right) * 100}{\sum_{i=1}^{n} Y_{i}^{o b s}}
$$

Em que: PBIAS é o desvio dos valores simulados (Ysim) em relação aos valores medidos yobs expresso em porcentagem.

Onde:

n: número de eventos;

Yobs: evento monitorado;

Y sim: evento simulado. 
Foram utilizadas as recomendações de Moriasi et al. (2007), que classifica o desempenho, por índice de eficiência estatística para modelagem de SWAT, conforme Tabela 01. Estes índices foram calculados tanto para os resultados das simulações calibradas, como os resultados das simulações não calibrados.

Tabela 1. Desempenho dos valores de COE e PBIAS.

\begin{tabular}{lll}
\hline Índice de eficiência Estatística & Resultados estatísticos de COE & Resultados estatísticos de PBIAS \\
\hline Muito Bom & $0.75<$ COE $\leq 1.00$ & PBIAS $< \pm 10$ \\
\hline Bom & $0.60<$ COE $\leq 0.75$ & $\pm 10 \leq$ PBIAS $< \pm 15$ \\
\hline Satisfatório & $0.50<$ COE $\leq 0.60$ & $\pm 15 \leq$ PBIAS $< \pm 25$ \\
\hline Insatisfatório & COE $\leq 0.50$ & PBIAS $> \pm 25$ \\
\hline
\end{tabular}

Fonte: Adaptado de Moriasi et al. (2007).

\section{RESULTADOS E DISCUSSÃO}

Com relação à área de drenagem da Bacia do Rio Tapajós, o SWAT estimou em 475983,31 km² sua área de abrangência e subdividiu em 255 sub-bacias, conforme apresentado na Figura 4. A correta delimitação das sub-bacias de um projeto possibilita extrair melhores resultados a partir da criação dos HRU's pela junção dos mapas de solo, declividade e cobertura do solo (ARNOLD, 1998). Subbacias minúsculas podem mascarar resultados e comprometer o bom funcionamento do modelo. Nesse trabalho foram criados $2.807 \mathrm{HRUS}$, possibilitando com isso analisar as áreas de forma mais detalhada. Os outlets, que são os pontos que definem o final de cada sub-bacia, três deles foram definidos a partir dos pontos fluviométricos inseridos manualmente no modelo, para que fosse possível realizar a comparação das vazões observadas e os simulados.

Nas Figuras 5 são apresentadas as vazões simuladas sem calibração e com calibração, sendo que nas Figuras 5A, 5B e 5C foram usadas como entradas os parâmetros climáticos GPCP, e nas Figuras 5D, 5E e 5F as entradas climáticas usadas foram o INMET, para o período de 1999 a 2002, para as sub-bacias 20, 41 e 172. 
Figura 5. Dados plotados de vazão observadas, vazão simulada sem calibração e com calibração das estações fluviométricas Acará do Tapajós (figura 5A), Jardim Ouro (figura 5B) e Fazenda Tratex (figura 5C) tendo como entradas climáticas GPCP; e as estações fluviométricas Acará do Tapajós (figura 5D), Jardim Ouro (figura 5E) e Fazenda Tratex (figura 5F) com dados climáticos INMET.
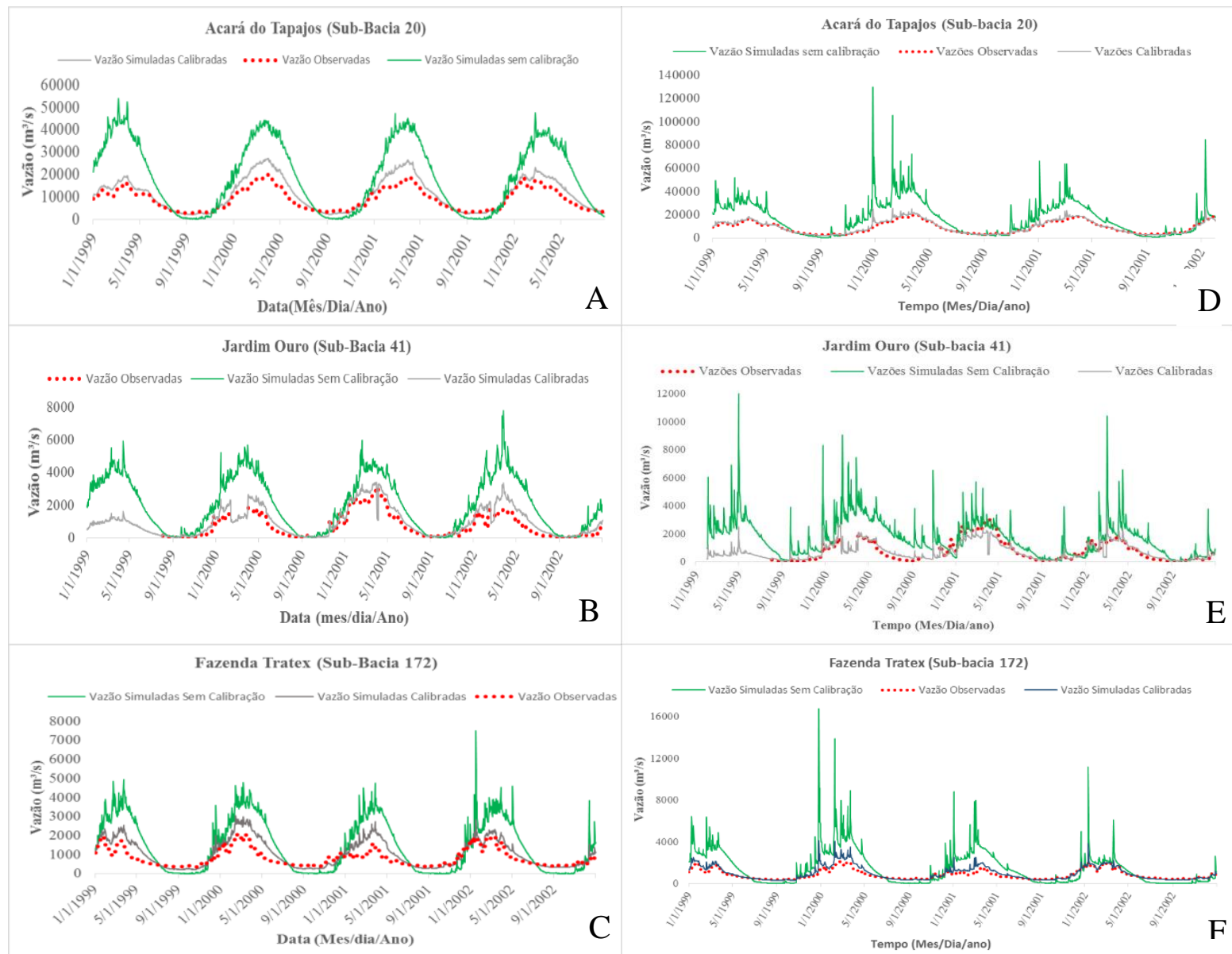

Fazenda Tratex (Sub-bacia 172)

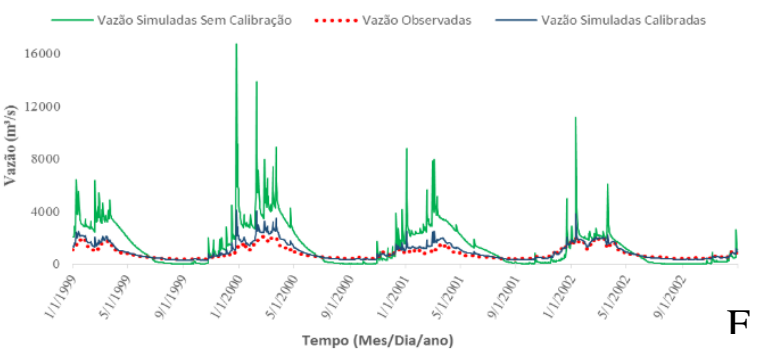

Fonte: Elaborado pelo autor (2017).

As simulações com SWAT sem o SWAT, como Ferrigo et al. (2011), Salles calibração, mesmo com as diferentes entradas climáticas, seguiram a mesma tendência das vazões observadas na subbacia 20, sub-bacia 41 e sub-bacia172, entretanto, os picos das vazões foram superestimados. Esse fenômeno já foi relatado em vários outros trabalhos com (2012) e Castro (2013). Também deve ser acrescentado que os valores superestimados na modelagem aqui apresentados, possuem maior discrepância no período chuvoso. Tal fato pode ser atribuído ao baixo nível piezométrico em que os rios se 
encontram no início desse período. Desta forma, as chuvas intensas iniciais são armazenadas nos rios, a fim de que os mesmos encham, só após atingir o equilíbrio que estes liberam vazões maiores, de acordo com a chuva.

As análises estatísticas destes resultados estão apresentadas na Tabela 2. para as sub-bacias 20, 41 e 172 os índices COE e PBIAS indicam que as vazões simuladas são insatisfatórias, considerando ambas as entradas climáticas, nas simulações sem calibração.

Tabela 2. Índices estatísticos das estações no modelo de SWAT

\begin{tabular}{|c|c|c|c|}
\hline \multicolumn{4}{|c|}{ Simulações com dados climáticos GPCP } \\
\hline \multicolumn{4}{|c|}{ Sem Calibração } \\
\hline Índice & Acará do Tapajós & Jardim Ouro & Fazenda Tratex \\
\hline Estatístico & Sub-bacia 20 & Sub-bacia 41 & Sub-bacia 172 \\
\hline $\mathrm{COE}$ & -6.81 & -3.05 & -5.93 \\
\hline PBIAS & $105.35 \%$ & $146.30 \%$ & $72.88 \%$ \\
\hline \multicolumn{4}{|c|}{ Com Calibração } \\
\hline $\mathrm{COE}$ & 0.56 & 0.68 & 0.37 \\
\hline PBIAS & $24.70 \%$ & $38.20 \%$ & $25.20 \%$ \\
\hline \multicolumn{4}{|c|}{ Simulações com dados climáticos INMET } \\
\hline \multicolumn{4}{|c|}{ Sem Calibração } \\
\hline Índice & Acará do Tapajós & Jardim Ouro & Fazenda Tratex \\
\hline Estatístico & Sub-bacia 20 & Sub-bacia 41 & Sub-bacia 172 \\
\hline COE & -3.14 & -1.07 & -7.62 \\
\hline PBIAS & $59.40 \%$ & $84.68 \%$ & $59.44 \%$ \\
\hline \multicolumn{4}{|c|}{ Com Calibração } \\
\hline COE & 0.81 & 0.86 & 0.79 \\
\hline PBIAS & $12.00 \%$ & $-1.38 \%$ & $12.90 \%$ \\
\hline
\end{tabular}

Fonte: Elaborado pelo autor (2017).

Avaliando de forma mais detalhada os valores informados pelo SWAT sem calibração, têm-se que a origem do problema é a superestimava do escoamento superficial. Nesta simulação, o volume do escoamento representou 
cerca 9,10\% do total precipitado, quando, na verdade, esse valor não deveria ultrapassar os 3\% (SILVA, 2015; OLIVEIRA, 1999; LIMA, 2013). O fator que mais contribui para a ocorrência desse fenômeno é o valor da Curva-Número, que difere muito dos solos americanos (para os quais o SWAT é inicialmente parametrizado) e os brasileiros (CARVALHO, 2014). Os solos brasileiros são muito antigos e intemperizados, sendo, por isso, profundos e muito permeáveis. Nesse cenário, a fim de refletir a realidade local, o valor inicial do parâmetro CN2 deve ser diminuído no processo de calibração (CARVALHO, 2014).

\section{CONCLUSÃO}

O índice de COE e PBIAS, utilizando como dados de entrada e parâmetros climáticos do INMET, demonstraram resultados calibrados bons e muito bons, segundo classificação proposta por Moriasi (2007). Assim, o modelo realizou a previsão do comportamento da vazão adequadamente. Estes resultados mostram que o modelo foi corretamente alinhado, interpretando as variáveis e, consequentemente, representando o comportamento hidrológico da bacia do rio Tapajós.

\section{REFERÊNCIAS}

ARAÚJO, R. C. Efeitos do desmatamento sobre o clico hidrológico: uma comparação entre a Bacia do Curuá - Una e a Bacia do Rio Uraim, Pará, 2010, f 123, (Tese Doutorado), Universidade Federal do Pará, Núcleo de Altos Estudos Amazônicos, Programa de PósGraduação em Desenvolvimento Sustentável do Trópico Úmido, Belém, 2010.

ARNOLD, J. G.; MUTTIAH, R. S.; SRINIVASAN, R.; ALLEN, P. M. Regional estimation of base flow and groundwater recharge in Upper Mississippi river basin. Journal of Hydrology, Amsterdam, v.227, n.1/4, p. 21-40, jan. 2000.

BALDISSERA, G. C. Aplicabilidade do modelo de simulação hidrológica swat (soil and water assessment tool), para a bacia hidrográfica do rio Cuiabá/MT. Mato Grosso, 2005, 148 f. (Dissertação de Mestrado) - Universidade Federal do Mato Grosso, Instituto de Ciências Exatas e da Terra, Programa de Pós-Graduação em Física e Meio Ambiente, Cuiabá, 2005.

CARVALHO F. H. Uso do modelo SWAT na estimativa da vazão e da produção de sedimentos em bacia agrícola do cerrado brasileiro. Brasília, 2014, f152. Dissertação (Mestrado) - Universidade de Brasília, Faculdade de Agronomia e Medicina Veterinária, Brasília, 2014.

CASTRO, K. B. Avaliação do modelo SWAT na simulação da vazão em bacia agrícola do cerrado intensamente monitorada. 2013. 141 f. (Dissertação de 
Mestrado) - Instituto de Geociências Universidade de Brasília, Brasília/DF. 2013.

COLLISCHONN, B.; COLLISCHONN, W.; TUCCl, C. E. M. Daily hydrological modeling in the Amazon basinusing TRMM rainfall estimates. Journal of Hydrology, v. 360, p. 207-216, 2008.

COE, M.T., COSTA, M.H., SOARES FILHO, B.S. The influence of historical and potential future deforestation on the stream flow of the Amazon River - Land surface processes and atmospheric feedbacks. Journal of Hydrology, v. 369, p.165-174, Mai., 2009.

$\operatorname{costA}, M . H$., et al. Climate change in Amazonia caused by soybean cropland expansion, as compared to caused by pastureland expansion. Geophysical Research Letters, v. 34, n. 7, p 1-4, 2007.

DURAES, M. F. Caracterização e avaliação do estresse hidrológico da bacia do rio Paraopeba, por meio de simulação chuva-vazão de cenários atuais e prospectivos de ocupação e uso do solo utilizando um modelo hidrológico distribuído. Minas Gerais, 2010, f 147, (Dissertação de mestrado), Universidade Federal de Minas Gerais, Belo Horizonte, MG, 2010.

FERRIGO, S.; MINOTI, R.T.; ROIG, H.; KOIDE, S. Análise do modelo SWAT na simulação de produção de sedimentos quando calibrado unicamente para vazão em uma pequena bacia hidrográfica rural. In: ENCONTRO NACIONAL DE ENGENHARIA DE SEDIMENTOS, 2012. Anais... Foz do Iguaçu, p 1091-1102, 2012.

FERRIGO, S.; MINOTTI, R.; KOIDE S. Utilização do modelo SWAT na estimativa de produção de sedimentos decorrentes de diferentes cenários de uso do solo na bacia do córrego Capão Comprido no Distrito Federal. In: SIMPÓSIO BRASILEIRO DE RECURSOS HÍDRICOS, 19., 2011. Anais... Maceió, 2011.

LIBOS, M.I.P.C. Modelagem da poluição não pontual na Bacia do Rio Cuiabá baseada em geoprocessamento. Rio de Janeiro, 2002, $296 f$. Dissertação (Mestrado em Engenharia Civil) - COPPE, Universidade Federal do Rio de Janeiro/RJ.

LIMA, J.E.F.W. Desenvolvimento de base de dados de solos para a aplicação do modelo SWAT em bacia do bioma Cerrado. In: SIMPÓSIO BRASILEIRO DE RECURSOS HÍDRICOS, 20., Maceió, 2013. Anais... Maceió, 2013. 8p.

MORIASI, D.N., ARNOLD, J.G., VAN LIEW, M.W., BINGNER, R.L., HARMEL, R.D., VEITH, T.L. Model evaluation guidelines for systematic quantification of accuracy in watershed simulations. Transaction softhe ASABE, v. 50, n. 3, p. 885-900. 2007.

NASH, J. E.; SUTCLIFFE, J. V. River flow forecasting through conceptual models. Part 1: a discussion of principles. Journal of Hydrological, v. 10, n. 3, p. 282-290, 1970.

OLIVEIRA, M. Q. C. Impacto de mudanças no uso do solo nas características hidrossedimentológicas da bacia hidrográfica do rio Joanes e sua repercussão na zona costeira, Bahia, 1999, 201 f. (Dissertação de Mestrado em Geologia) - Universidade Federal da Bahia/BA 1999.

PONTES, P. R. M.; COLLISCHONN, W.; FAN, F. M.; PAIVA, R. C. D.; BUARQUE, D. C. Modelagem hidrológica e hidráulica de grande escala com propagação inercial 
de vazões / Hydrologic and hydraulic large-scale modeling with inertialflowrouting. Brazilian Journal Water Resource, v. 20, n. 4, p. 888-904, 2015.

PONTES, S.H.N.; FERNANDES, I.V. Atualização do método das isozonas para a região amazônica. In: ENCONTRO DE INICIAÇÃO CIENTÍFICA E PÓSGRADUAÇÃO DO ITA, 8., 2002. Anais... São Paulo, SP, 2002.

RIBEIRO, C. B. M.; MOHANTY, B. P.; BRESSIANI, D. A.; FERNANDES, J. G.; FILHO, O. C. R. Parameterization of physical and climatic characteristics in the Amazon basin for hydrological simulation with SWAT model. In: INTERNATIONAL SWAT CONFERENCE, 11., 2014, Pernambuco. Anais... Pernambuco, 2014.

RODRIGUES, E. L.; ELMIRO, M. A. T.; JACOBI, C. M.; LAMOUNIER, W. L. Aplicação do modelo SWAT na avaliação do consumo de água em áreas de florestas plantadas na bacia do rio Pará, alto São Francisco, em Minas Gerais, Sociedade \& Natureza, Uberlândia, v. 27, n. 3, p. 485-500, Set./Dez., 2015.

RUHOFF, A. L. Gerenciamento de recursos hídricos em bacias hidrográficas: modelagem ambiental com a simulação de cenários presenvacionistas. Santa Maria, 2005, 105 f, (Dissertação de mestrado), UFSM, Santa Maria, 2004.

SALLES, L. A. Calibração e validação do modelo SWAT para a predição de vazões na bacia do ribeirão Pipiripau. (Dissertação de mestrado), Brasília, DF, 2012, 114 f Universidade de Brasília, Brasília, DF, 2012.

SANTOS, I. Desenvolvimento de modelo de avaliação da qualidade de água e transporte de sedimentos para pequenas e médias centrais elétricas: modelagem da erosão e transporte de sedimentos. Curitiba - PR: Instituto de Tecnologia para o Desenvolvimento (LACTEC), 2005. 91 p.

SAXTON, K.E., RAWLS, W.J. Soil Water Characteristic Estimates by Textureand Organic Matter for Hydrologic Solutions. Soil Science Society of Agronomy Journal, v. 70, n. 5, p. 1569-1578, 2006.

SILVA, L. R. S. Análise de incertezas e avaliação dos fatores influentes no desempenho de modelos de simulação de bacias hidrográficas. Tese (Doutorado) - Universidade de Brasília, Programa de Pós-Graduação em Tecnologia Ambiental e Recursos Hídricos, Brasília, 2013.

SILVA, L.A. SWAT applications in eastern Amazonia: A case study of the Acará Mirim and Bujaru river basin in State of Pará, Brazil, 2015, USP/EMBRAPA, p.2.

SOUZA, R. M. de; SANTOS, I. dos; KOBIYAMA, M. O modelo SWAT como ferramenta para a gestão de recursos hídricos: avaliação de vazões de outorga na bacia hidrográfica do altíssimo rio negro. In: SIMPÓSIO BRASILEIRO DE RECURSOS HÍDRICOS, XVIII Cidade, Anais. Porto Alegre, RS: Associação Brasileira de Recursos Hídricos, 2009.

SRINIVASAN, R.; ARNOLD, J. G. Integration of the basin-scale water quality model with GIS. Jornal of the American Water Resources Bulletin, v. 30, p. 453-462, 1994.

WAGENER, $T$.; WHEATER, $H_{\text {.; }}$ GUPTA, $H$. $V$. Rainfall-Runoff modeling in gauged and ungauged catchments. 1.ed. Londres: Imperial College, 2004. 332 p. 